\title{
Long-Term Response and Remission with Pixantrone in Patients with Relapsed or Refractory Aggressive Non-Hodgkin Lymphoma: Post-Hoc Analysis of the Multicenter, Open-Label, Randomized PIX301 Trial
}

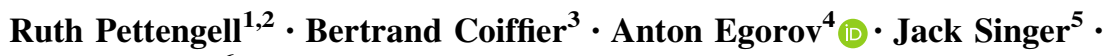 \\ Lilia Sivcheva ${ }^{6}$
}

Published online: 21 March 2018

(c) The Author(s) 2018. This article is an open access publication

\begin{abstract}
Background Pixantrone is recommended in relapsed and refractory non-Hodgkin lymphoma (NHL) or heavily pretreated NHL patients. Its conditional approval in Europe was based on results from the open-label, randomized, phase 3 PIX301 study, comparing pixantrone monotherapy with physician's choice of treatment in 140 patients with relapsed or refractory aggressive NHL.

Methods This post-hoc analysis of the PIX301 study investigated possible correlations between patient characteristics and clinical response in 17 patients $(24 \%)$ treated with pixantrone who achieved a complete response (CR) or an unconfirmed complete response $(\mathrm{CRu})$ at study end.

Results These patients (10 male and 7 female) had a median age of 61 (range 41-75) years, and the most common diagnoses were diffuse large B-cell lymphoma $(n=10)$ and transformed indolent lymphoma $(n=4)$. Most had received two prior lines of therapy $(n=12)$. There was wide variation in the time from diagnosis to study entry (219-4777 days). Among the 17 patients who achieved a CR/CRu with pixantrone, 6 had stable or progressive disease as a response to their last regimen, 7 had a
\end{abstract}

Ruth Pettengell

rpetteng@sgul.ac.uk

1 St George's Hospital, London, UK

2 Institute of Medical and Biomedical Education, St George's University of London, London, UK

3 Centre Hospitalier Lyon Sud, Pierre-Bénite, Lyon, France

4 Institut de Recherches Internationales Servier (IRIS), Suresnes, France

5 Cell Therapeutics Inc. (CTI), Seattle, WA, USA

6 MHAT "Hristo Botev", Vratsa, Bulgaria partial response, and 4 had a $\mathrm{CR} / \mathrm{CRu}$. Four patients from the pixantrone group survived without progression for more than 400 days. Prior response to previous therapies did not appear to affect long-term response to pixantrone. Conclusions These observations suggest that pixantrone monotherapy in patients with multiply relapsed or refractory aggressive NHL who had received at least two prior therapies can be associated with durable responses and long-term remission, and this may be unrelated to the clinical response to the last therapy.

\section{Key Points}

In the PIX301 study, patients with relapsed/ refractory non-Hodgkin lymphoma who achieved a complete response with pixantrone had various responses to prior therapies (complete or partial response, stable or progressive disease).

Four patients receiving pixantrone who had previously had a complete or partial response $(n=1$ each) or progressive disease $(n=2)$ with prior therapies, were free from progression for more than 400 days.

These results suggest that pixantrone monotherapy can induce durable responses and long-term remission in some patients with relapsed/refractory non-Hodgkin lymphoma. These responses appear unrelated to the response to prior therapies. 


\section{Introduction}

Current management of relapsed/refractory non-Hodgkin lymphoma (NHL) remains an unmet medical need, due to a poor prognostic outlook and lack of treatment options [1]. First-line therapy for aggressive B-cell NHL is an anthracycline-based regimen in combination with rituximab, such as R-CHOP (rituximab, cyclophosphamide, doxorubicin, vincristine and prednisolone) [2-4]. Complete response rates with R-CHOP of around $80 \%$ have been reported, with a 5-year progression-free survival (PFS) of approximately $50 \%$ [5]. However, up to $25 \%$ of patients inevitably relapse [6].

Due to the risk of cardiotoxicity with increasing cumulative anthracycline dose, repeated use of anthracyclines is limited in patients with relapsed/refractory disease $[7,8]$. Newer agents are few, and include ibrutinib for relapsed/refractory mantle-cell lymphoma [9] and idelalisib for refractory follicular lymphoma [10]. Pixantrone, an agent that belongs to the aza-anthracenedione family $[1,11]$, is a recommended option in multiply relapsed and refractory disease or heavily pretreated patients with NHL $[3,12]$. Pixantrone's molecular structure is different from anthracyclines $[1,13,14]$. The differences in its structure result in a unique mechanism of action, notably with a lesser potential for iron binding and therefore less cardiotoxicity compared with anthracyclines $[1,11]$.

Pixantrone has conditional approval in Europe [11], based on results from the open-label, randomized phase 3 PIX301 study [15, 16], where significantly more patients who received pixantrone than comparator (physician's choice of treatment) achieved a complete (CR) or an unconfirmed complete response $(\mathrm{CRu})$ and a higher overall response rate (ORR) [15]; median PFS was also significantly prolonged in the pixantrone arm. It is indicated as monotherapy for the treatment of adult patients with multiply relapsed or refractory aggressive B-cell NHL [11]. To complete post-authorization measures, a confirmatory phase 3 study (PIX306) is underway [15]. Study recruitment is complete and results from the primary efficacy endpoint analysis are expected in 2018.

There were several cases of long-term remission in the PIX301 study, including a case of a very durable partial response (PR) with pixantrone in a patient with relapsed diffuse large B-cell lymphoma (DLBCL) at study entry (see Box 1), in addition to some patients with durable CRs. In the current post-hoc analysis of data from the PIX301 trial, we explored possible correlations between patient characteristics and clinical response in these cases.

\section{Methods}

\subsection{PIX301 Trial Study Design}

The design and methods of the phase 3 PIX301 study have been described previously [15]. Briefly, PIX301 was a multicenter, open-label, randomized trial [15]. A total of 140 patients were enrolled in 66 hospital centers in Europe, India, Russia, South America, UK and USA between October 2004 and March 2008. All patients provided written informed consent, and local ethical approval was obtained in all centers. The study was registered at ClinicalTrials.gov (NCT00088530).

Enrolled patients were those with aggressive de novo or transformed NHL, who had relapsed or were refractory to at least two previous multi-agent chemotherapy regimens, with previous therapy including at least one standard anthracycline-based regimen with a response of at least 24 weeks. During the course of the study, the protocol was amended to exclude patients with no previous treatment with rituximab in countries where it was commercially available $[15,16]$. Patients also had to have a European Cooperative Oncology Group performance status of $0-2$, measurable disease, a left ventricular ejection fraction of at least $50 \%$, adequate bone marrow and organ function and no persisting toxicities from previous lines of treatment. Patients were excluded if they had received a cumulative dose of doxorubicin or equivalent exceeding $450 \mathrm{mg} / \mathrm{m}^{2}$, or if they had New York Heart Association grade 3 or 4 cardiovascular abnormalities [15].

Patients were randomized (1:1) to receive pixantrone dimaleate $85 \mathrm{mg} / \mathrm{m}^{2}$ (equivalent to $50 \mathrm{mg} / \mathrm{m}^{2}$ in the base formulation approved by the European regulatory authorities) by intravenous infusion over $1 \mathrm{~h}$ on days 1,8 and 15 every 28 days for up to six cycles or a comparator agent [15]. The choice of comparator was left to the treating physician, but suitable choices included vinorelbine, oxaliplatin, ifosfamide, etoposide, mitoxantrone, gemcitabine or rituximab administered at prespecified standard doses and schedules. Randomization was achieved by an interactive voice response system and was stratified by region (USA vs Western Europe vs the rest of the world), International Prognostic Index score (0 or 1 vs $\geq 2$ ), and previous autologous stem-cell transplant (SCT) [yes vs no] [15]. Patients were followed for 18 months after the last treatment administration for disease progression and survival [15].

\subsection{Post-Hoc Study Design}

This post-hoc analysis included only patients with a CR or $\mathrm{CRu}$ to pixantrone during the PIX301 study in the 
intention-to-treat population. Potential relationships between patient characteristics, previous therapies and response to pixantrone were assessed. Additionally, patient characteristics of long-term responders to pixantrone were explored.

\subsection{Statistical Methods}

This study used descriptive statistics only. Values are presented as means (standard deviation [SD]), medians (range) or numbers (percentages).

\section{Results}

\subsection{Patients}

Overall, 17 patients treated with pixantrone in the intention-to-treat population achieved a CR/CRu in the PIX301 trial (i.e. $24 \%$ of the pixantrone treatment arm). Demographics and clinical characteristics of these patients are shown in Table 1. The median age of these patients was 61 (range 41-75) years, and there were slightly more male

Table 1 Demographics and characteristics of the 17 patients with a $\mathrm{CR} / \mathrm{CRu}$ with pixantrone

\begin{tabular}{lc}
\hline Characteristic & Value \\
\hline Age, years & $61(41-75)$ \\
Median (range) & $61.4(9.6)$ \\
Mean (SD) & \\
Sex, $n(\%)$ & $10(58.8)$ \\
Male & $7(41.2)$ \\
Female & \\
Diagnosis, $n(\%)$ & $10(58.8)$ \\
DLBCL & $4(23.5)$ \\
Transformed indolent lymphoma & $1(5.9)$ \\
Peripheral T-cell lymphoma NOS & $1(5.9)$ \\
Anaplastic large cell lymphoma & $1(5.9)$ \\
Follicular lymphoma & $1(5.9)$ \\
Prior CT scan, $n(\%)$ & \\
Previous lines of therapy, $n(\%)$ & $12(70.6)$ \\
2 & $4(23.5)$ \\
3 & $1(5.9)$ \\
$\geq 4$ &
\end{tabular}

$C R$ complete response, $C R u$ unconfirmed complete response, $C T$ computed tomography, DLBCL diffuse large B-cell lymphoma, NOS not otherwise specified, $S D$ standard deviation than female patients (10 vs 7 patients). The most common diagnoses were DLBCL (10 patients) and transformed indolent lymphoma (4 patients). Most patients had received two prior lines of therapy (12 patients, $71 \%$ ). Of note, there was wide variation in the time from diagnosis to study entry (219-4777 days). Two protocol violations were subsequently identified, where two patients had received monotherapy in the treatment line prior to randomization (Table 2).

\subsection{Exploration of Patient Characteristics, Previous Therapy and Response to Pixantrone}

Among the 17 patients who achieved a CR/CRu with pixantrone, 6 had stable disease or progressive disease (PD) as a response to their prior regimen, 7 had a partial response (PR), and 4 had a CR/CRu (Table 2). The achievement of a durable response with pixantrone therefore appeared to be independent of the type of response to prior therapy (CR, PR, or stable disease).

\subsection{Characteristics of Patients with Durable Responses to Pixantrone}

Four patients survived without progression for more than 400 days (Table 2 and Fig. 1). These patients had a duration of response to pixantrone of $679,633,623$ and 448 days; respective ages were $75,60,63$ and 58 years; respective histologies were DLBCL, DLBCL, transformed indolent lymphoma and transformed indolent lymphoma; and respective numbers of prior lines of therapy were two, two, two and three. The patient with the longest response duration to pixantrone was the only one of the four patients to have received prior rituximab treatment. Response to prior chemotherapy did not appear to affect the long-term response to pixantrone. Respective last therapies (response; duration of treatment) prior to pixantrone were R-CHOP (CR; 67 days); DHAP (cytarabine, dexamethasone, cisplatin) [PD; 43 days]; ICE (ifosfamide, carboplatin, etoposide) [PD; 43 days]; and ESHAP (etoposide, methylprednisolone, cytarabine, cisplatin) [PR; 96 days].

\section{Discussion}

At the end of treatment in the main PIX301 study [15], significantly more patients who received pixantrone achieved a CR/CRu (20.0 vs $5.7 \% ; p=0.021)$ and an ORR (37.1 vs $14.3 \% ; p=0.003$ ) than did those who received a comparator drug. Median PFS was also significantly prolonged in the pixantrone treatment group [5.3 vs 2.6 months; hazard ratio (HR) $0.60 ; 95 \%$ CI $0.42,0.86$; $p=0.005]$. A post-hoc analysis of data from a subgroup of 
Table 2 Previous therapy received by the 17 patients with a CR/CRu

\begin{tabular}{|c|c|c|c|c|c|}
\hline $\begin{array}{l}\text { Patient } \\
\text { number }\end{array}$ & Prior regimen & $\begin{array}{l}\text { Duration of prior } \\
\text { regimen, days }\end{array}$ & $\begin{array}{l}\text { Response to } \\
\text { last regimen }\end{array}$ & $\begin{array}{l}\text { Time between end of last regimen } \\
\text { and initiation of pixantrone, days }\end{array}$ & $\begin{array}{l}\text { Duration of response } \\
\text { to pixantrone, days }\end{array}$ \\
\hline 1 & $\begin{array}{l}\text { Rituximab plus etoposide, } \\
\text { carmustine, } \\
\text { methylprednisolone }\end{array}$ & 114 & PR & 506 & 121 \\
\hline 2 & R-CHOP & 67 & CR & 637 & 679 \\
\hline 3 & Chlorambucil $^{\mathrm{a}}$ & 99 & Stable disease & 238 & 18 \\
\hline 4 & R-DHAP & 202 & Stable disease & 512 & 151 \\
\hline 5 & $\begin{array}{l}\text { Cytarabine, dexamethasone, } \\
\text { ifosfamide }\end{array}$ & 3 & PD & 53 & 213 \\
\hline 6 & ICE & 117 & $\mathrm{CR}$ & 249 & 56 \\
\hline 7 & ICE & 28 & PD & 54 & 623 \\
\hline 8 & $\begin{array}{l}\text { Cytarabine, dexamethasone, } \\
\text { cisplatin }\end{array}$ & 43 & PD & 226 & 633 \\
\hline 9 & CVP & 90 & PR & 2043 & $1^{\mathrm{b}}$ \\
\hline 10 & CVP & 47 & PR & 1108 & 63 \\
\hline 11 & ESHAP & 96 & PR & 275 & 448 \\
\hline 12 & $\begin{array}{l}\text { Rituximab plus cytarabine, } \\
\text { dexamethasone, carboplatin }\end{array}$ & 115 & $\mathrm{CRu}$ & 602 & 308 \\
\hline 13 & R-CHOP & 107 & CR & 354 & 166 \\
\hline 14 & Dexamethasone, gemcitabine & 229 & PR & 335 & 80 \\
\hline 15 & CVP & 109 & PR & 622 & 82 \\
\hline 16 & R-ESHAP & 105 & PR & 171 & 333 \\
\hline 17 & Cyclophosphamide $^{\mathrm{a}}$ & 5 & Stable disease & 50 & 291 \\
\hline
\end{tabular}

$C R$ complete response, $C R u$ unconfirmed complete response, $C V P$ cyclophosphamide, vincristine, prednisone, ESHAP cytarabine, dexamethasone, cisplatin, etoposide, ICE ifosfamide, carboplatin, etoposide, $P D$ progressive disease, $P R$ partial response, $R$-CHOP rituximab plus cyclophosphamide, doxorubicin, vincristine, prednisone, $R$-DHAP rituximab plus dexamethasone, high-dose cytarabine, cisplatin, $R$-ESHAP rituximab plus etoposide, methylprednisolone, high-dose cytarabine, cisplatin

${ }^{a}$ Protocol violation due to monotherapy as previous therapy

${ }^{\mathrm{b}}$ Patient censored without a true progression or death

Fig. 1 Duration of best response (from onset of a $\mathrm{CR}$ / $\mathrm{CRu}$ to last confirmation of nonprogression). $C R$ complete response, $C R u$ unconfirmed complete response. Asterisk: Censored (patient did not progress or die)

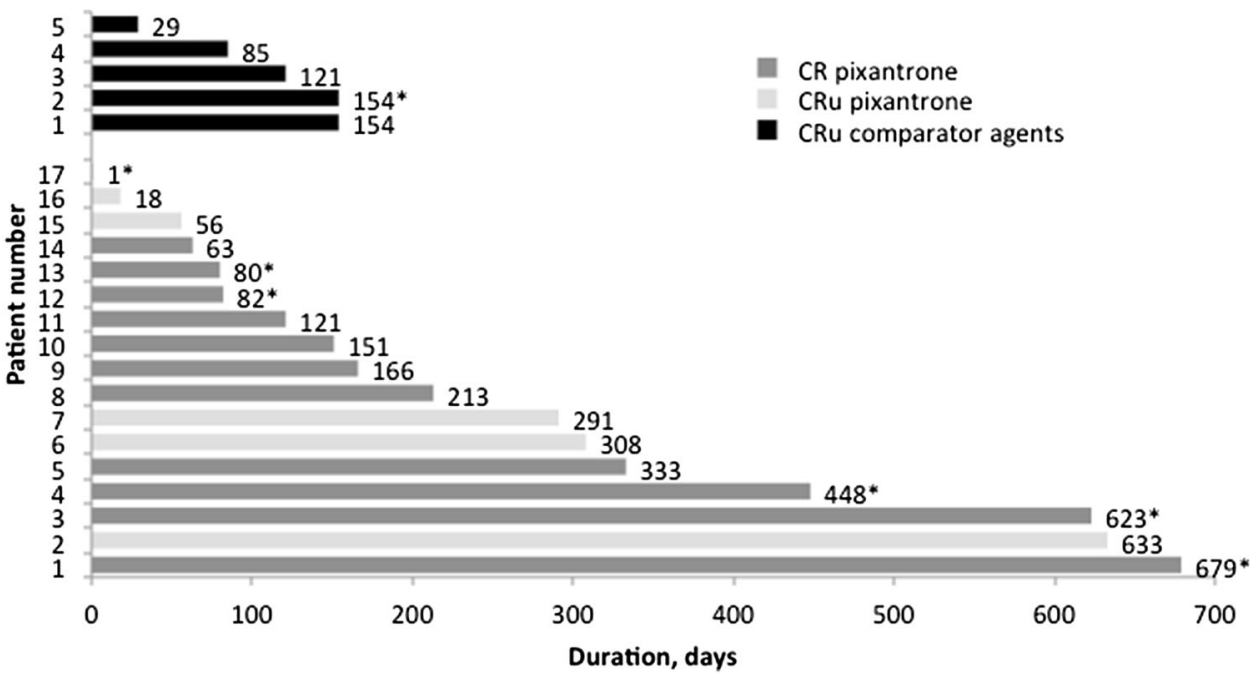

patients in the study with histologically confirmed relapsed or refractory aggressive B-cell NHL also demonstrated a significantly improved $\mathrm{CR} / \mathrm{CRu}(23.1$ vs $5.1 \% ; p=0.047)$ and ORR (43.6 vs $12.8 \% ; p=0.005)$ with third- or fourthline pixantrone treatment compared with comparator agents [16]. Clinical response was especially improved 
among those patients who had received previous rituximab treatment, with an improved CR (30.0 vs $5.6 \%$; $p=0.093)$, ORR (45.0 vs $11.1 \% ; p=0.033)$, and median PFS (5.4 vs 2.8 months; HR 0.52; 95\% CI 0.26, 1.04) with pixantrone versus comparator agents.

The results of the present analysis build on previous results and suggest that it is possible to obtain a longlasting response with pixantrone. Also, the potential for a durable response to pixantrone appears to be independent of whether the best response to prior therapy was a CR, PR, stable disease, or progressive disease. Of note, our results indicate that it is possible to get a response with later lines of NHL treatment, even with monotherapy, which is important because monotherapy is typically associated with lower rates of toxicities than multi-agent regimens.

Our observations are in line with those in a phase 2 study of pixantrone monotherapy in patients with relapsed, aggressive NHL, in which several patients had very long CRs that lasted more than 1 year [17]. In this study, two female patients with stage II DLBCL and stage IV mantle cell lymphoma experienced CRs lasting $17+$ and 15.2 months, respectively. This study also included one patient (male; aged 66 years) with stage IV transformed follicular lymphoma who experienced a PR lasting 24+ months.

There are several advantages of long-term responses in this setting, where treatment options are limited. Treatment is usually of palliative intent, and patients are often unable to tolerate combination regimens because of factors including comorbidities, age, poor performance status and cardiotoxicity from previous anthracycline therapy [1]. Therefore, monotherapies with good tolerability and longterm efficacy are sorely needed [1]. Pixantrone was designed to maintain efficacy while reducing the risk of cardiotoxicity. It is non-cross-resistant with anthracyclines and generally well tolerated with a manageable toxicity profile, making it a useful treatment option [13, 18].

Case studies have also indicated that pixantrone may be useful in the routine clinic setting, as a bridge to autologous $\mathrm{SCT}$, or to induce a CR following relapse after allogenic SCT [19, 20]. One patient with DLBCL achieved a CR with third-line pixantrone monotherapy plus a single dose of rituximab and benefited from long-term remission after consolidation with high-dose chemotherapy and autologous SCT [19]. Another patient with DLBCL who relapsed after allogeneic SCT achieved a CR with pixantrone without any considerable side effects [20].

Patients with long-term responses clearly have a tumor that is chemosensitive to pixantrone. Intercalation of pixantrone into DNA results in successive rounds of aberrant mitosis, ultimately leading to cell death [13]. In contrast to anthracyclines, pixantrone is only a weak inhibitor of topoisomerase II, and while it directly alkylates DNA, forming stable DNA adducts and cross-strand breaks $[1,11]$, the main way pixantrone causes cell death is to impair the fidelity of mitosis without triggering the DNA damage response or mitotic checkpoint activation. While it is clear that pixantrone has a mechanism of action that is unique from anthracyclines, the specific mechanisms involved in the long-term responses seen among patients receiving pixantrone in PIX301 are unknown.

Our study has several limitations. First, the descriptive nature of the analysis is an obvious limitation, as is the small sample size, which prevents firm conclusions from being drawn. Additionally, there was no biopsy or positron emission tomography (PET) scan performed at the time of relapse and study entry for the clinical case presented in Box 1, which is because PET scans and imaging were not standard practice at the time of study entry. The clinical case received etoposide and corticosteroids in the second line, which could be regarded as an inadequate second-line treatment, but this did not appear to negatively affect the outcome.

\section{Conclusion}

Our observations imply that treatment with pixantrone monotherapy in patients with multiply relapsed or refractory aggressive NHL who had received at least two prior therapies can be associated with durable responses and long-term remission. Our results suggest that a durable response with pixantrone can be achieved irrespective of the clinical response to last therapy, but this needs to be confirmed in a larger number of patients.

\section{Boxed Case Report}

Box 1 Clinical case of long-term remission with pixantrone

A 55-year-old male patient presented with enlarged inguinal, para-iliac, and bronchopulmonary lymph nodes, sweating and weight loss in November 2006. In December 2006, an inguinal lymph node biopsy was performed, and histological and immunohistochemical findings showed diffuse large B-cell lymphoma (DLBCL), stage IIIB. By June 2007, the patient had achieved a partial response (PR) after six cycles of $\mathrm{CHOP}$ as first-line treatment. In December 2007, the patient presented with enlarged inguinal and para-iliac lymph nodes, indicating disease progression. The patient then received second-line therapy with etoposide and corticosteroids. There was further disease progression in January 2008. In February 2008, the patient signed an informed consent to participate in PIX301. Computed tomography (CT) assessment revealed Ann Arbor stage III with enlarged 
bronchopulmonary and para-iliac lymph nodes. The diagnosis was confirmed by blinded central histological review, as per protocol. The bone marrow biopsy revealed no bone marrow involvement; the patient had an Eastern Cooperative Oncology Group performance status of 1 and an International Prognostic Index of 1, and no history of previous or concomitant diseases. He was randomly allocated to pixantrone in March 2008 and started therapy with $85 \mathrm{mg} / \mathrm{m}^{2}$ pixantrone dimaleate (which corresponds to $50 \mathrm{mg} / \mathrm{m}^{2}$ in pixantrone's base form), given on days 1 , 8 and 15 of each 28-day cycle for six cycles. Treatment was completed in August 2008 without any missed or reduced doses. Response to therapy was monitored using CT every 8 weeks, as well as 28 days after the end of treatment. The patient achieved a PR at the end of treatment. Electrocardiographic measurements, troponin $\mathrm{T}$, multigated acquisition scan and echocardiogram were used to assess possible cardiotoxicity; all were within normal limits. No adverse events were observed during treatment, except for relatively long-lasting skin discoloration (over 6 months after the end of the therapy). After the end of treatment (August 2008), CT monitoring was performed regularly $(4,6,8,10,12,14$ and 18 months) until June 2010 and annually thereafter. No disease progression was observed. The patient's last visit was in January 2017; he was asymptomatic, with no evidence of recurrent disease.

Acknowledgements Medical writing assistance with the preparation of this manuscript was provided by Andrea Bothwell and Marie Cheeseman on behalf of Springer Healthcare Communications. This medical writing assistance was funded by Servier.

\section{Compliance with Ethical Standards}

Conflict of interest RP has received honoraria from CTI and Servier. LS has received research funding from CTI as a trial investigator. JS is an employee of CTI. AE is an employee of Servier. BC declares no conflicts of interest.

Ethics approval All procedures performed in this study were in accordance with the ethical standards of the responsible institutional research committee of each center involved and with the $1964 \mathrm{Hel}-$ sinki Declaration and its later amendments.

Funding This study was funded by Servier and CTI. Article processing charges were funded by Servier.

Informed consent Informed consent was obtained from all individual participants included in the study.

Author contributions Ruth Pettengell, Bertrand Coiffier and Jack Singer contributed to study design, enrolled patients, and read and approved drafts. Lilia Sivcheva enrolled patients and provided the case report and reviewed and approved the manuscript. Anton Egorov performed the analysis of the PIX301 clinical study report for the scope of this publication and read and provided comments to successive drafts and approved the final version.
Open Access This article is distributed under the terms of the Creative Commons Attribution-NonCommercial 4.0 International License (http://creativecommons.org/licenses/by-nc/4.0/), which permits any noncommercial use, distribution, and reproduction in any medium, provided you give appropriate credit to the original author(s) and the source, provide a link to the Creative Commons license, and indicate if changes were made.

\section{References}

1. Volpetti S, Zaja F, Fanin R. Pixantrone for the treatment of adult patients with relapsed or refractory aggressive non-Hodgkin B-cell lymphomas. Onco Targets Ther. 2014;7:865-72.

2. Dreyling M, Ghielmini M, Rule S, et al. Newly diagnosed and relapsed follicular lymphoma: ESMO Clinical Practice Guidelines for diagnosis, treatment and follow-up. Ann Oncol. 2016;27(suppl 5):v83-90.

3. Tilly H, Gomes da Silva M, Vitolo U, et al. Diffuse large B-cell lymphoma (DLBCL): ESMO Clinical Practice Guidelines for diagnosis, treatment and follow-up. Ann Oncol. 2015;26(suppl 5):v116-25.

4. Vitolo U, Seymour JF, Martelli M, et al. Extranodal diffuse large B-cell lymphoma (DLBCL) and primary mediastinal B-cell lymphoma: ESMO Clinical Practice Guidelines for diagnosis, treatment and follow-up. Ann Oncol. 2016;27(suppl 5):v91-102.

5. Molina A. A decade of rituximab: improving survival outcomes in non-Hodgkin's lymphoma. Annu Rev Med. 2008;59:237-50.

6. Larouche JF, Berger F, Chassagne-Clement C, et al. Lymphoma recurrence 5 years or later following diffuse large B-cell lymphoma: clinical characteristics and outcome. J Clin Oncol. 2010;28(12):2094-100.

7. Fanous I, Dillon P. Cancer treatment-related cardiac toxicity: prevention, assessment and management. Med Oncol. 2016;33(8):84.

8. Swain SM, Whaley FS, Ewer MS. Congestive heart failure in patients treated with doxorubicin: a retrospective analysis of three trials. Cancer. 2003;97(11):2869-79.

9. Janssen-Cilag. Imbruvica: summary of product characteristics. 2014. http://www.ema.europa.eu/docs/en_GB/document_library/ EPAR_-_Product_Information/human/003791/WC500177775. pdf. Accessed 7 Feb 2018.

10. Gilead Sciences. Zydelig: summary of product characteristics. 2014. http://www.ema.europa.eu/docs/en_GB/document_library/ EPAR__Product_Information/human/003843/WC500175377. pdf. Accessed 7 Feb 2018.

11. CTI Life Sciences Limited. Pixuvri: summary of product characteristics. 2017. http://www.ema.europa.eu/docs/en_GB/ document_library/EPAR_-_Product_Information/human/002055/ WC500127968.pdf. Accessed 10 July 2017.

12. Zinzani PL, Corradini P, Martelli M, et al. Critical concepts, practice recommendations, and research perspectives of pixantrone therapy in non-Hodgkin lymphoma: a SIE, SIES, and GITMO consensus paper. Eur J Haematol. 2016;97(6):554-61.

13. Beeharry N, Di Rora AG, Smith MR, Yen TJ. Pixantrone induces cell death through mitotic perturbations and subsequent aberrant cell divisions. Cancer Biol Ther. 2015;16(9):1397-406.

14. Salvatorelli E, Menna P, Paz OG, et al. The novel anthracenedione, pixantrone, lacks redox activity and inhibits doxorubicinol formation in human myocardium: insight to explain the cardiac safety of pixantrone in doxorubicin-treated patients. J Pharmacol Exp Ther. 2013;344(2):467-78.

15. Pettengell R, Coiffier B, Narayanan G, et al. Pixantrone dimaleate versus other chemotherapeutic agents as a single-agent salvage treatment in patients with relapsed or refractory aggressive non- 
Hodgkin lymphoma: a phase 3, multicentre, open-label, randomised trial. Lancet Oncol. 2012;13(7):696-706.

16. Pettengell R, Sebban C, Zinzani PL, et al. Monotherapy with pixantrone in histologically confirmed relapsed or refractory aggressive B-cell non-Hodgkin lymphoma: post-hoc analyses from a phase III trial. Br J Haematol. 2016;174(5):692-9.

17. Borchmann P, Morschhauser F, Parry A, et al. Phase-II study of the new aza-anthracenedione, BBR 2778, in patients with relapsed aggressive non-Hodgkin's lymphomas. Haematologica. 2003;88(8):888-94.

18. Keating GM. Pixantrone: a review in relapsed or refractory aggressive non-Hodgkin's lymphoma. Drugs. 2016;76(16):1579-86.
19. Appio L, Landoni C, La Targia M, et al. Single-agent pixantrone as a bridge to autologous stem cell transplantation in a patient with refractory diffuse large B-cell lymphoma. Chemotherapy. 2017;62(3):187-91.

20. Malaspina F, Pellegrini C, Casadei B, Argnani L, Zinzani PL. Impressive response to pixantrone after allogeneic transplant in a multiple relapsed diffuse large B-cell lymphoma. Acta Haematol. 2017;137(4):191-4. 